\title{
Light Time Effects in Pulsating Variables and Brown Dwarfs
}

\author{
Jiang Shi Yang \\ National Astronomical Observatories of China, Chinese Academy of \\ Sciences, Datun Road 20A, Beijing 100012, China
}

\begin{abstract}
Dwarf cepheids DY Her, AD CMi, and BE Lyn show light time effects, which may be caused by orbital motion due to a low mass unseen companion. Their mass is probably between 0.015 to $0.07 \mathrm{M}_{\odot}$, so they may be brown dwarfs.
\end{abstract}

\section{Introduction}

A brown dwarf (BD) is a low mass object with a mass between 0.07 and $0.015 \mathrm{M}_{\odot}$, so that they cannot have hydrogen fusion energy support for their emission. At first astronomers assumed that a good place to look for very faint objects would be close to known stars. There are two kinds of influences we can see. One is the small amplitude periodical variation in the proper motion of normal star. Another is the low amplitude periodical variation in the radial velocity of normal star. Other astronomers pursued a different strategy that took advantage of the fact that BDs are brightest when they are young. The best place to look for young objects is in young star clusters. Astronomers found still more BDs using another search technique: looking for them at random locations in the sky. They are low surface temperature objects, so the most efficient survey way is to use infrared detectors. There are 3 such surveys: DENIS, 2MASS and Sloan Digital Sky Survey. In the future the Chinese LAMOST will do the same thing with much higher sensitivity and fainter limiting magnitude.

\section{Other new ways}

For pulsating variables or eclipsing binaries, we can observe another kind of influence, the low amplitude periodical variation of time of light maximum or minimum (Deeg et al. 2000; Guinan and Ribas 2001). For many pulsating variables, the direct observation shows it is a single star. But its time of light maximum does not repeated regularly with constant period. Sometimes, the time differences between two successive times of light maximum is longer, sometimes is shorter. If this kind of variation is periodic, that means this pulsating star is moving around on an orbit surrounding the common mass center of the two stars. One is the pulsating variable another is its unseen low-mass companion. If the mass function $f\left(m_{2}\right)=\left(m_{2} \sin i\right)^{3} /\left(m_{1}+m_{2}\right)^{2}=(\Delta t c)^{3} /\left(p_{\text {orbit }}\right)^{2}$ is very small, especially if fluctuation of time of light maximum ( $t$ is very small, the mass of unseen companion $m_{2}$ will be very small. So it is highly probably 
a brown dwarf. Here $\mathrm{m} 1$ is the mass of the pulsating star, $\mathrm{c}$ is light speed, $\mathrm{i}$ is inclination angle of the orbit with the line of sight. As we can measure $t$ with quite high accuracy, this method is easier than to measure the periodical variation of proper motion or radial velocity.

\section{Our results}

We present here three examples:

- AD CMi: Fu and Jiang (1996) found $P_{\text {orbit }}=30 \mathrm{yr}, \Delta t c=0.483 \mathrm{AU}$, so the mass function is about 0.000125 . $\mathrm{AD} \mathrm{CMi}$ is a high amplitude population I $\delta$ Scuti type variable, its mass should be between 1.5 to $2.5 \mathrm{M}_{\odot}$. From the HIPPARCOS parallax, we know AD CMi is below the main sequence, so its mass must be quite small. If $m_{1}=1.5 \mathrm{M}_{\odot}, m_{2} \sin i$ $=0.054 \mathrm{M}_{\odot}$. The $v \sin i$ of AD CMi is $20 \mathrm{~km} / \mathrm{s}$. The pulsating amplitude in $V$ band is 0.3 magnitude. So its rotating velocity $\mathrm{v}$ must be very close to $20 \mathrm{~km} / \mathrm{s}$. Then the $\sin i$ of its equator must be very close to 1 , and the $\sin i$ of its orbit should be very close to 1 . The unseen companion mass should be close to $0.05 \mathrm{M}_{\odot}$, thus a brown dwarf.

- DY Her: Pocs and Szeidl (2000) found $P_{\text {orbit }}=43 \mathrm{yr}, \Delta \mathrm{t} \mathrm{c}=0.22 \mathrm{AU}$, so $\mathrm{f}\left(m_{2}\right)=0.0000058$. If $m_{1}=2 \mathrm{M}_{\odot},\left(m_{2} \sin i\right) \leq 0.03 \mathrm{M}_{\odot}$. As its $v \sin i=20$ $\mathrm{km} / \mathrm{s}$, from its pulsating amplitude in $\mathrm{V}$ band of 0.51 mag., $\sin i$ should be very close to 1 , the unseen companion mass should be close to $0.03 \mathrm{M}_{\odot}$, thus a brown dwarf.

- BE Lyn: Kiss and Szatmary (1995) found $P_{\text {orbit }}=6.44 \mathrm{yr}, \Delta \mathrm{t} \mathrm{c}=0.12$ AU, so f $\left(m_{2}\right)=0.00004$. If $m_{1}=1.7 \mathrm{M}_{\odot}, m_{2}$ should be between 0.05 to $0.3 \mathrm{M} \odot$. As we do not know its $v \sin i$, the mass is not well determined. But if $i$ larger than 46 degrees, it must be a brown dwarf.

Among the ten dwarf Cepheids which have light time effects caused by unseen companion, two have $\mathrm{BD}$ masses, so it looks there is no BD desert at all in this sample. Of course, we haven't finally proved the BD nature yet for all 3 unseen companions. As for the extra-solar planets, all of the companions that we discovered so far have period shorter than 10 years. If we go to longer period, it may be no BD desert at all.

\section{References}

Deeg, H. et al. 2000, A\&A, 358, L5

Fu, J. and Jiang, S. 1996, IBVS, No. 4325

Guinan, E. F. and Ribas, I., 2001, ApJ, 546, L43

Kiss, L. L. and Szatmary, K., 1995, IBVS, No. 4166

Pocs, M. D. and Szeidl, B., 2000, IBVS, No. 4832 